\title{
Non-Linear Analysis of the Main Atrial Wave to Estimate Organization in Paroxysmal Atrial Fibrillation
}

\author{
R Alcaraz ${ }^{1}$, JJ Rieta $^{2}$ \\ ${ }^{1}$ Innovation in Bioengineering Research Group. University of Castilla-La Mancha, Cuenca, Spain \\ ${ }^{2}$ Biomedical Synergy. Valencia University of Technology, Valencia, Spain
}

\begin{abstract}
The analysis of spontaneous Atrial Fibrillation (paroxysmal $A F$ ) termination or maintenance could avoid unnecessary therapy and contribute to take the appropriate decisions on its management. The aim of this work is to study if an AF episode terminates spontaneously or not by analyzing the increase of atrial activity ( $A A)$ organization prior to AF termination. The organization varies as a consequence of the decrease in the number of reentries into the atrial tissue. The analysis was carried out noninvasively through the use of surface electrocardiogram (ECG) recordings. Sample entropy (SampEn) was selected as non-linear organization index. It was observed that noise and ventricular residues degrade AA organization estimation performance, therefore the use of selective filtering to get the main atrial wave (MAW) was necessary. Using the $M A W$ organization analysis, that is the signal produced by the fundamental atrial waveform, 46 out of 50 of the terminating and non-terminating analyzed AF episodes were correctly classified (92\%). Therefore, this method can provide a clear benefit for patients, doctors and health systems, since it avoids useless therapeutic intervention and minimises the risks for the patients.
\end{abstract}

\section{Introduction}

Atrial Fibrillation (AF) is a supraventricular arrhythmia characterized by uncoordinated atrial activation. $\mathrm{AF}$ occurs when the electrical impulses in the atria degenerate into a rapid chaotic pattern [1]. On the ECG, AF is described by the replacement of $\mathrm{P}$ waves by fibrillatory waves ( $f$ waves) that vary in size, shape, and timing, associated with an irregular ventricular response. Consequently, when AF occurs, a notably disorganized atrial activity (AA) can be observed [2].

Clinically speaking, AF is the most common cardiac arrhythmia, affecting $1 \%$ of the general population. Considering its prevalence with age, this arrhythmia affects up to $15 \%$ of the population older than 80 and has an in- cidence that doubles with each advancing decade. Paroxysmal (spontaneously terminated) AF is, by evidence, antecedent to persistent $\mathrm{AF}$, which requires a pharmacological or external electrical intervention (cardioversion) to allow its termination [1]. AF predisposes to thrombus formation within the atria that can cause stroke or any other thromboembolic events [3]. Thus, the prediction of AF termination, based on non-invasive techniques, can be of great clinical value in order to avoid useless therapeutic interventions and to minimice the associated risks.

The most widely accepted theory to explain AF is based on the continuous propagation of multiple wavelets (called reentries) wandering throughout the atria [1]. The number of simultaneous reentries depends on the refractory period, mass and conduction velocity along the atria. On the other hand, several studies have demonstrated a decrease in the number of reentries prior to $\mathrm{AF}$ termination, thus producing simpler wavefronts and $f$ waves evolve to $\mathrm{P}$ waves. In other words, the AA slightly evolves to a more organized pattern before AF termination [2]. This fact can be used to predict AF termination when the proper organization analysis tools are used.

Previous groups studied non-linear complexity indexes to characterize AA organization from the surface ECG, but their results did not revealed significative differences between terminating and non-termating AF episodes. The low signal to noise ratio was considered as the main reason to this unsuccessful result [4]. Therefore, a reduction of noise, ventricular residues, and any other nuisance signal could give positive results with non-linear analysis.

In the present work, the AA obtained from the surface ECG has been processed making use of selective filtering adapted to the peak atrial frequency. Next, the main atrial wave (MAW) was obtained. The MAW can be considered as the signal associated to the fundamental atrial waveform, its wavelength being the inverse of the AA main frequency [5]. The MAW organization has been estimated by means of sample entropy (SampEn), to discriminate between terminating and non-terminating AF episodes. This non-linear tool quantifies the regularity of a time series [6] 
and its use in this study is justified because (i) the nonlinearity, as necessary condition for a chaotic behavior, is present in the diseased heart with AF at cellular level and (ii) the electrical remodelling in $\mathrm{AF}$ is a far-from-linear process [7].

\section{Materials}

\subsection{Database}

The used database contained 50 one minute and two leads (II and V1) electrocardiogram (ECG) recordings, which were available in Physionet [8]. They were extracted from 24-hour Holter recordings from 50 different patients. The database included non-terminating AF episodes (group $\mathrm{N}$ ), which were observed to continue in AF for at least one hour following the end of the excerpt, and AF episodes terminating immediately after the end of the extracted segment (group T). Recordings were divided into a learning and a test set. Next, 10 labelled recordings of each group formed the learning set. An optimal threshold, which should allow to discern between terminating and non-terminating paroxysmal AF episodes, was defined making use of the proposed methodology together with the learning set. Finally, the test set was composed with the remaining 30 recordings.

\subsection{Data preprocessing}

The ECG recordings were preprocessed in order to reduce noise, nuisance interferences and improve later analysis. Firstly, baseline wander was removed making use of bidirectional high pass filtering with $0.5 \mathrm{~Hz}$ cutt-off frequency [9]. Secondly, high frequency noise was reduced with a eight order bidirectional IIR Chebyshev low pass filtering, whose cut-off frequency was $70 \mathrm{~Hz}$ [10]. Finally, powerline interference was removed through adaptive filtering, which preserves the ECG spectral information [11].

\section{Methods}

\subsection{Sample entropy}

Sample Entropy (SampEn) examines time series for similar epochs and assigns a non-negative number to the sequence, with larger values corresponding to more complexity or irregularity in the data [12]. Two input parameters, a run length $m$ and a tolerance window $r$, must be specified for SampEn to be computed. $\operatorname{SampEn}(m, r)$ is the negative logarithm of the conditional probability that two sequences similar during $m$ points remain similar at the next point, where self-matches are not included in calculating the probability. Thus, a lower value of SampEn also indicates more self-similarity in the time series. Sam$\mathrm{pEn}$ is largely independent on record length [6, 12]. A more detailed mathematical description can be found in [12].

Although $m$ and $r$ are critical in determining the outcome of SampEn, no guidelines exist for optimizing their values. Nevertheless, the $m$ and $r$ values suggested by Pincus are $m=1$ or $m=2$ and $r$ between 0.1 and 0.25 times the standard deviation of the original time serie [13].

\subsection{Main atrial wave derivation and classi- fication}

The proposed methodology to obtain the MAW is shown in Fig. 1. Firstly, cancellation of QRST waves from the ECG signals was performed, obtaining a raw approximation to the MAW. Though a variety of QRST cancellation techniques exist, the average QRST template cancellation method was used, since only two leads were available [14]. Next, the power spectral density (PSD) of the residual signal was calculated using Welch Periodogram. A Hamming window of 4096 points in length, a 50\% overlapping between adjacent windowed sections and a 8192-points Fast Fourier Transform (FFT) were used as computational parameters. The frequency with the largest amplitude within the 3-9 Hz range was selected as the dominant atrial frequency. Finally, the MAW was obtained applying a selective filtering to the AA signal centered around the dominant atrial frequency.

To prevent distortion, a linear phase FIR filter was used [15]. Chebyshev approximation was preferred because all the filter parameters can be suitably fitted and minimum ripple in the pass and stop bands were needed. Therefore, a high order filter should be used, such as the Kaiser approximation marks [16]:

$$
M=\frac{-20 \log _{10}\left(\sqrt{\delta_{1} \delta_{2}}\right)-13}{14.6 \Delta f}+1
$$

where $M$ is the filter order, $\delta_{1}$ and $\delta_{2}$ are the pass and stop bands ripple, respectively, and $\Delta f$ is the transition bandwidth between bands. A selective filter must have $\delta_{1}$ and $\delta_{2}$ lower than $0.5 \%$ of the gain and $\Delta f$ lower than $0.01 \mathrm{~Hz}$, thereby its order must be greater than 250 .

The selected filter bandwidth should be lower than $6 \mathrm{~Hz}$ because the typical frequency range of the AA is around 3-9 Hz [17]. In our experiments, the best results were obtained with a $3 \mathrm{~Hz}$ bandwidth and 768 filter coefficients. The MAW organization results obtained through the application of SampEn to the learning set, defined empirically the optimum threshold (Th) that, later, will allow the test set classification into terminating and non-terminating AF episodes. 


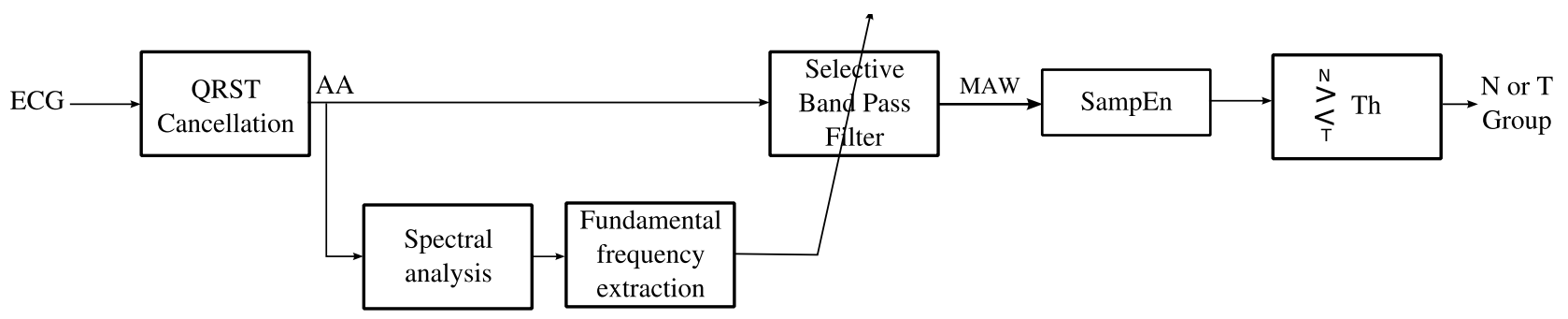

Figure 1. Block diagram describing the proposed methodology. Firstly, the ventricular activity is removed from the input ECG to obtain the AA. Next, a low bandwidth selective filter is applied to the atrial signal. The filter is centered around the dominant atrial frequency. SampEn is computed on the resulting signal and threshold compared in order to decide if the episode terminates.

\section{Results}

The proposed methodology was applied to the learning set and $100 \%$ sensivity and $90 \%$ specificity were obtained, see Fig. 2(a). The Receiver Operating Characteristic (ROC) curve provided 0.084115 as optimum SampEn discrimination threshold between terminating and nonterminating AF sets.

Fig. 2(b) shows the SampEn values for the 20 learning signals together with the mean and standard deviation values for each group. Note that all non-terminating and 9 out of 10 terminating recordings (95\% of the learning signals) can be correctly discriminated.

Making use of the aforementioned SamEn threshold, 27 out of 30 test signals $(90 \%)$ were correctly classified, see Fig. 2(c). Therefore, the AF behaviour of 46 out of 50 recordings $(92 \%)$ were correctly predicted through the MAW organization analysis.

The SampEn mean value and standard deviation for all terminating and non-terminating AF recordings, and the statistic significance obtained by the t-student are shown in Table 1. Note that the terminating episodes present lower SampEn values than the non-terminating ones. Indeed, both paroxysmal AF groups are statistically distinguishable, given that statistic significance is notably lower than 0.01. Finally, remark that the obtained SampEn values are quite low, because of the MAW is a notably regular wave.

Table 1. Mean value and standard deviation for $\mathrm{T}$ and $\mathrm{N}$ sets and the t-student statistical significance.

\begin{tabular}{c|cc}
\hline \hline & N Group & T Group \\
\hline Mean \pm Std & $0.1047 \pm 0.01352$ & $0.0747 \pm 0.0156$ \\
$\mathbf{p}$ & \multicolumn{2}{|c}{0.00000000245} \\
\hline \hline
\end{tabular}

\section{Discussion and conclusions}

By analyzing with SampEn main atrial wave organization prior to spontaneous AF termination, $92 \%$ of the ter- minating and non-terminating AF episodes were correctly classified. In order to obtain the MAW, the AA signal was firstly extracted. Several ways to extract the AA from surface ECG recordings have been presented. The latest presented methods take advantage of multiple lead information by solving the blind source separation problem [18]. Other methods are based on ventricular activity cancellation, such as QRST template matching and subtraction [14] or spatiotemporal QRST cancellation [17]. These methods obtain the (so called) remainder ECG that consists mainly of the AA. In this study, the QRST cancellation method was used [14].

Bearing in mind that the mean or median beat cannot represent each individual beat accurately, since QRST morphology is affected by respiration, patient movement, etc., QRST residuals and noise are often present in the remainder ECG [19]. These nuisance signals degrade AA organization estimation provoking very poor results when direct AA organization analysis was applied [4] and, consequently, MAW obtention was necessary.

This method can provide a clear benefit for patients, doctors and health systems, since it avoids useless therapeutic intervention (cardioversion) and minimises the risks for the patients. However, off-line analysis and high computational burden are limitations that should be refined.

\section{Acknowledgements}

This work was partly supported by the projects 20070086 from the R+D+i Vice-rectorate of the Valencia University of Technology, GV06/299 from Consellería de Empresa, Universidad y Ciencia de la Generalitat Valenciana and TEC2007-64884 from the Spanish Ministry of Education and Science.

\section{References}

[1] Fuster V, Ryden LE, Asinger RW, et al. ACC/AHA/ESC 2006 guidelines for the management of patients with atrial fibrillation: a report of the American College of Cardiol- 


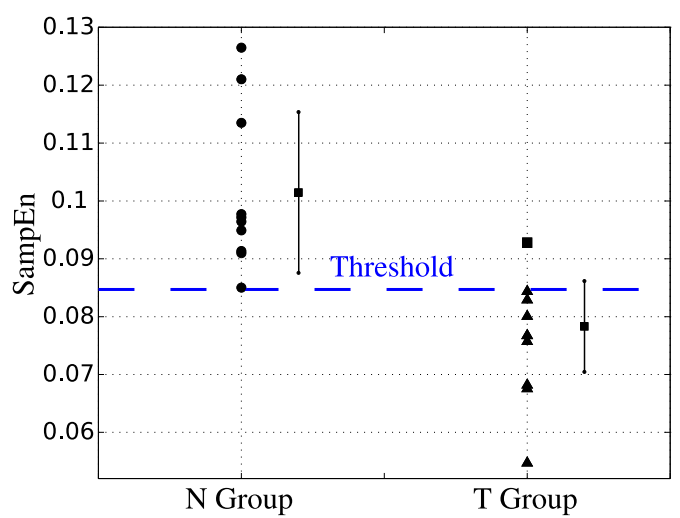

(a)

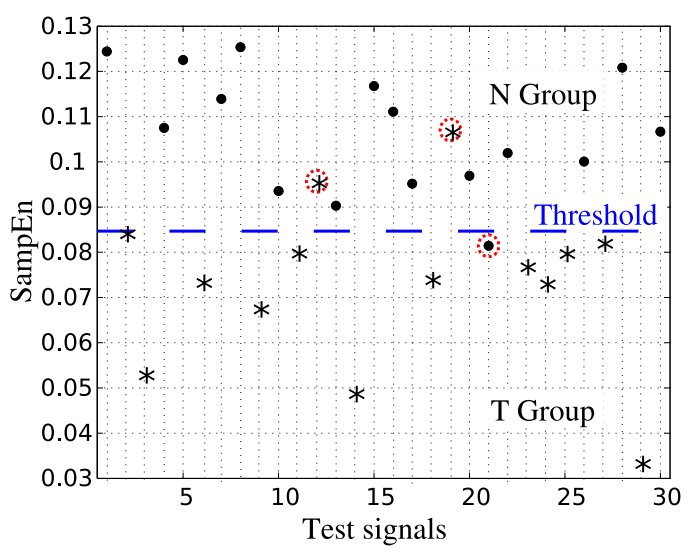

(b)

Figure 2. Classification into non-terminating and terminating AF for the recordings in (a) learning set and (b) test set.

ogy/American Heart Association Task Force on practice guidelines and the European Society of Cardiology committee for practice guidelines developed in collaboration with th european heart rhythm association and the heart rhythm society. Europace 2006;8(9):651-745.

[2] Bollmann A, Lombardi F. Electrocardiology of atrial fibrillation - Current knowledge and future challenges. IEEE Engineering in Medicine and Biology Magazine 2006; 25(6):15-23.

[3] Blomstrom-Lundqvist C, Scheinman MM, Aliot EM, Alpert JS, et. al. ACC/AHA/ESC guidelines for the management of patients with supraventricular arrhythmias. European Heart Journal 2003;24(20):1857-1897.

[4] Nilsson F, Stridh M, Bollmann A, Sörnmo L. Predicting spontaneous termination of atrial fibrillation using the surface ECG. Medical Engineering Physics 2006;8:802-808.

[5] Holm M, Pehrson S, Ingemansson M, Sörnmo L, Jahansson R, Sandhall L, Sunemark M, Smideberg B, Olsson C, Olsson $\mathrm{S}$. Non-invasive assessment of the atrial cycle length during atrial fibrillation in man: introducing, validating and illustrating a new ECG method. Cardiovasc Res 1998; 38(1):69-81.

[6] Pincus SM. Approximate entropy as a measure of system complexity. in Proc Natl Acad Sci USA 1991;88(6):22972301.

[7] Bollmann A. Quantification of electrical remodeling in human atrial fibrillation. Cardiovasc Res 2000;47:207-209.

[8] Goldberger AL, Amaral LA, Glass L, Hausdorff JM, Ivanov PC, Mark RG, Mietus JE, Moody GB, Peng CK, Stanley HE. Physiobank, physiotoolkit, and physionet: components of a new research resource for complex physiologic signals. Circulation 2000;101(23):e215-e220.

[9] Dotsinsky I, Stoyanov T. Optimization of bi-directional digital filtering for drift suppression in electrocardiogram signals. J Med Eng Technol 2004;28(4):178-180.

[10] Sun Y, Chan K, Krishnan SM. ECG signal conditioning by morphological filtering. Comput Biol Med 2002; 32(6):465-479.

[11] Ferdjallah M, Barr RE. Adaptive digital notch filter design on the unit-circle for the removal of powerline noise from biomedical signals. IEEE Transaction on Biomedical Engineering 1994;42(6):529-536.

[12] Richman JS, Moorman JR. Physiological time series analysis using approximate entropy and sample entropy. Am J Physiol 2000;278(6):H2039-H2049.

[13] Pincus SM. Assessing serial irregularity and its implications for health. Annals of the New York Academic of Sciences 2001;954:245-267.

[14] Slocum J, Sahakian A, Swiryn S. Diagnosis of atrial fibrillation from surface electrocardiograms based on computerdetected atrial activity. Journal of Electrocardiology 1992; 25(1):1-8.

[15] Sörnmo L, Laguna P. Bioelectrical Signal Processing in Cardiac and Neurological Applications. Elsevier Academic Press, 2005. ISBN 0-12-437552-9.

[16] Rabiner LR, McClellan JH, Parks TW. FIR digital filter design techniques using weighted chebyshev approximation. Proc IEEE 1975;63:595-610.

[17] Stridh M, Sörnmo L. Spatiotemporal QRST cancellation techniques for analysis of atrial fibrillation. IEEE Trans Biomed Eng 2001;48(1):105-111.

[18] Rieta JJ, Castells F, Sánchez C, Zarzoso V, Millet J. Atrial activity extraction for atrial fibrillation analysis using blind source separation. IEEE Trans Biomed Eng 2004; 51(7):1176-1186.

[19] Petrutiu S, Nijm GM, Angari HA, Swiryn S, Sahakian AV. Atrial fibrillation and waveform characterization - A time domain perspective in the surface ECG. IEEE Engineering in Medicine and Biology Magazine 2006;25(6):24-30.

Address for correspondence:

Raúl Alcaraz Martínez

E. U. Politécnica de Cuenca

Campus Universitario

16071 Cuenca (Spain)

raul.alcaraz@uclm.es 